\title{
Lighting conditions and optical filters effects on visual performance of speleologists exposed to cave environments
}

\author{
Carla Costa Lança ${ }^{1 *}$, Nádia Fernandes ${ }^{1}$, Ana Monteiro ${ }^{2}$, and Ana Almeida ${ }^{3,4}$ \\ ${ }^{1}$ Orthoptic Department, Lisbon School of Health Technology, Av. D. João II, Lote 4.69.01, 1990 - 096 Lisboa, Portugal \\ ${ }^{2}$ Environmental Health Department, Lisbon School of Health Technology, Av. D. João II, Lote 4.69.01, 1990 - 096 Lisboa, Portugal \\ ${ }^{3}$ Hospital Beatriz Angelo, Avenida Carlos Teixeira, 3, 2674-514 Loures, Portugal \\ ${ }^{4}$ Nova Medical School, Campo Mártires da Pátria. 130, 1169-056 Lisboa, Portugal
}

\begin{abstract}
The aim of this study is to evaluate lighting conditions and speleologists' visual performance using optical filters when exposed to the lighting conditions of cave environments. A crosssectional study was conducted. Twenty-three speleologists were submitted to an evaluation of visual function in a clinical lab. An examination of visual acuity, contrast sensitivity, stereoacuity and flashlight illuminance levels was also performed in 16 of the 23 speleologists at two caves deprived of natural lightning. Two organic filters (450 nm and $550 \mathrm{~nm}$ ) were used to compare visual function with and without filters. The mean age of the speleologists was $40.65( \pm 10.93)$ years. We detected $26.1 \%$ participants with visual impairment of which refractive error $(17.4 \%)$ was the major cause. In the cave environment the majority of the speleologists used a head flashlight with a mean illuminance of $451.0 \pm 305.7$ lux. Binocular visual acuity (BVA) was -0.05 \pm 0.15 LogMAR (20/18). BVA for distance without filter was not statistically different from BVA with $550 \mathrm{~nm}$ or $450 \mathrm{~nm}$ filters $(p=0.093$ ). Significant improved contrast sensitivity was observed with $450 \mathrm{~nm}$ filters for $6 \mathrm{cpd}(p=0.034)$ and $18 \mathrm{cpd}(p=0.026)$ spatial frequencies. There were no signs and symptoms of visual pathologies related to cave exposure. Illuminance levels were adequate to the majority of the activities performed. The enhancement in contrast sensitivity with filters could potentially improve tasks related with the activities performed in the cave.
\end{abstract}

Keywords: $\quad$ speleologists; visual function; optical filters; contrast sensitivity; illuminance levels Received 13 June 2015; Revised 22 October 2015; Accepted 3 November 2015

Citation: Lança C.C., Fernandes N., Monteiro A. and Almeida A., 2016. Lighting conditions and optical filters effects on visual performance of speleologists exposed to cave environments. International Journal of Speleology, 45 (1), 27-33. Tampa, FL (USA) ISSN 0392-6672 http://dx.doi.org/10.5038/1827-806X.45.1.1933

\section{INTRODUCTION}

Work related conditions in a cave such as exposure to heat, chemicals, dust, and poor lighting could influence the integrity of the visual system and predispose the eye to diseases that eventually affect vision (OvenseriOgbomo et al., 2012). Poor lighting conditions cause a variety of symptoms of visual discomfort and may increase the risk of accidents (Veitch, 2001; Van Bommel, 2006; Reinhold \& Tint, 2009; Pais \& Melo, 2011). Visual discomfort results in signs and symptoms such as eyestrain, blurred vision, visual irritability, headaches, muscle aches and stress (Boyce et al., 1997; Kerkhof, 1999; Van Bommel, 2006; Pais \& Melo, 2011). There are also other symptoms caused by the lack of lightning: tired eyes and watery and itchy eyes. Other specific disorders include degeneration of vision sharpness (blurred and diplopia) and slowness in changing focus (Woodside \& Kocurek, 1997; Blehm et., 2005; Reinhold \& Tint, 2009).
Contrast sensitivity and visual acuity are fundamental descriptors of the human visual system playing a key role in the quality of vision (Kohnen et al., 2005; Kang et al., 2009). Good visual acuity is crucial for several professions (Johnson \& Casson, 1995) regarding safety purposes. Visual performance is critical in miner's ability to judge the speed or direction of a machine (Reyes et al., 2013) and for cavers to explore, map or perform research in caves. Visual acuity typically measured under optimal viewing conditions with appropriate refractive correction will be altered by different environmental conditions and refractive properties in the work environment (Johnson \& Casson, 1995).

There are some professional activities involving visual tasks with resolution of detail that must be performed under conditions of reduced illumination and contrast. Speleologists perform their activity in demanding visual conditions of low illumination, as 
cave environment has similar conditions to night vision. LED light systems could help to improve de lighting conditions. The visual system is able to operate effectively from starlight to bright sunlight; over a change in illumination by more than a factor of $10^{11}$ (Stockman \& Sharpe, 2006). However, changes in visual function occur under reduced illumination: reductions in visual acuity in central and peripheral locations, as well as reduced contrast sensitivity for all spatial frequencies (Wood \& Owens, 2005). Visual contrast sensitivity is an indicator of visual patterndetection ability for stimuli of various sizes. Visual stimuli encountered in everyday life activities are often of much lower contrast, due to various conditions such as inclement weather or darkness (Zavod, 2004).

Filters are currently used by eye care practitioners to assist people with low vision in maximising use of residual vision, improve visual function, control glare and improve orientation and mobility skills (Eperjesi et al., 2002). It could also be used in some tasks to improve contrast sensitivity, selectively absorbing light on the short wavelength end of the visible spectrum, where the rods are most sensitive, and transmitting light on the long end of the spectrum (Thomas et al., 2010).

The visual function during speleological activities has not been documented. The aim of this study is to evaluate lighting conditions and speleologists' visual performance using optical filters when exposed to the lighting conditions of cave environments. The specific objectives of this study are to (1) evaluate visual function of speleologists who were directly involved in caving and/or are exposed to the cave environment, (2) evaluate lighting conditions for those doing exploration and research in caves, (3) evaluate visual performance in the cave environment, and (4) evaluate visual performance with filters in the cave environment.

\section{METHODS}

A cross-sectional study was conducted between December 2013 and January 2014. Twenty-three Portuguese speleologists participated in the present study. Examination procedures were thoroughly explained and informed consent was obtained prior to participation. Lisbon School of Health Technology (ESTeSL) Ethics Committee has approved the protocol for the research project. This study adhered to the tenets of the Declaration of Helsinki in 1995 (as revised in Edinburgh 2000).

A questionnaire was administered to participants to describe their socio demographic data, previous and current work history, detailed medical and ocular history, current use and type of medication, the use of protective eye wear, lighting conditions, activities performed, accidents, time and length of stay in the cave.

\section{Visual function tests}

All participants underwent a visual examination conducted by 2 Orthoptists at ESTeSL Clinical Orthoptic Laboratory and in two Portuguese caves lacking of natural lightning. All subjects who normally wore corrective lenses were asked to wear them during vision testing. The first step involved a visual function examination at ESTeSL. Twentythree volunteer speleologists were submitted to an evaluation of visual acuity, contrast sensitivity, stereoacuity, refractive error (auto-refraction), intraocular pressure (tonometry), ocular alignment and near convergence point. The second step involved a visual function examination (visual acuity, contrast sensitivity and stereoacuity) in the cave environment. Sixteen speleologists agreed to spend half a day in the cave and evaluations of flashlight levels were also undertaken. In this step seven subjects were excluded from the study because they were not available during the study period.

Two organic filters (450 $\mathrm{nm}$ and $550 \mathrm{~nm}$ ) were used to measure and compare visual function (distance visual acuity, near visual acuity and contrast sensitivity) with and without filters in the cave environment.

The visual acuity and contrast sensitivity tests were administered monocularly to each eye in the lab (an eye occluder was held over one eye while the other eye was tested) to identify visual impairment and binocularly in the cave environment to evaluate functional vision.

Distance visual acuity (VA) was assessed in mesopic conditions in the lab at a distance of $2.5 \mathrm{~m}$ with an Early Treatment Diabetic Retinopathy Study (ETDRS) CSV 1000 Vector Vision Chart. Visual acuity was recorded as the last line on which at least 3 of the 5 letters were identified correctly. Pinhole acuity was assessed in eyes presenting VA higher than 0.1 LogMAR.

Near visual acuity was assessed in photopic conditions at a distance of $40 \mathrm{~cm}$ with an ETDRS GoodLite chart. Visual acuity was recorded as the last line on which at least 3 of the 5 letters were identified correctly.

Contrast sensitivity was assessed in mesopic conditions with the Vector Vision - CSV 1000 E. The test contained a matrix of circles filled with sinusoidal gratings (dark and light bars). Spatial frequency $(3,6,12$, and 18 cycles/degree) increased from top to bottom, and contrast decreased from left to right. The grating bars were oriented vertically. The contrast level of the last test patch correctly identified on each row was recorded as the contrast sensitivity score for that row (log units). The procedure was repeated for each row in descending order. Distance visual acuity of 0.5 LogMAR or better was a criterion to perform this test in order to avoid confounding the results by excessive optical refraction error (Hudnell et al., 2001).

Ocular alignment was assessed only in the lab with a cover test (CT) at distance and near $(6 \mathrm{~m}$ and $33 \mathrm{~cm}$, respectively) to test the presence of heterotropias and heterophorias. The CT was performed with the head held straight and a black paddle occluder as a cover. Detailed fixation objects were used as targets. Manifest strabismus was defined as constant or intermittent tropia of any magnitude at distance or near fixation (Friedman et al., 2009). A prism cover test was employed to assess the magnitude of the deviation present. 
Near convergence point $(\mathrm{NCP})$ : the assessment of NCP was conducted with the Royal Air Force (RAF) ruler only in the lab.

Stereoacuity was assessed with a Randot ${ }^{\circledR}$ at $40 \mathrm{~cm}$. A card with superimposed images of circles was shown to the speleologist and the ability to detect the elevation of the circle's above the plane of the card indicated stereopsis level in a range from $800 "$ to $40 "$.

Objective refraction and tonometry: Subjects were also submitted to a screening for refractive error with non-cycloplegic auto-refraction using a Gr-21 autorefractometer and measurements of intraocular pressure with a Canon Tonometer TX-10.

Filters: Two filters consisting of organic material with $450 \mathrm{~nm}$ and $550 \mathrm{~nm}$ were used (ML filters optical solutions). The filters used in this study absorb blue light and could be used by people with normal vision to improve visual performance in many tasks under different light level's condition because their properties help to improve best contrast achieved. Transmittance levels were measured with a spectrophotometer (Thermo Scientific Evolution 300). Three aspects of visual function were analysed, with and without filters, in the cave environment: distance visual acuity, near visual acuity and contrast sensitivity.

\section{Lighting levels measurements}

All speleologists use portable lighting sources for their cave activities. Illumination was emitted by a Petzl LED flashlight on the helmet, head, or hand (up to 60 lumens). The flashlight could be adjusted to a maximum of four different levels of light intensity (1 to 4): the lower light intensity was named level 1 and the higher light intensity is referred to as 4 . The flashlight also had a beam diffuser, which could be used to transform the focused light beam into a wide light beam. All flashlight levels were measured with and without the filter diffuser. In the evaluations of the illuminance levels, the time chosen for the determination of these levels were not fixed a priori. In each point, the measurements were carried out for a sufficient time in order to stabilize the light levels values. These measurements were conducted at a distance of half a meter and at a height of one meter. The illuminance measurements were made using a silicon diode from a Gossen lux meter (model Mavolux 5032C). The lux meter incorporates color correction and its spectral response was tested for the human eye.

\section{Statistical analysis}

Data was analysed using the Statistical Package for Social Sciences (SPSS, v. 19). Appropriate descriptive and inferential statistics were used to present the results of the study. A $p$ value of less than 0.05 was accepted as significant. Significant differences in contrast sensitivity of the right and left eyes were analysed using the related-samples Wilcoxon. Significant differences in performance between the no filter condition and the two filters (450/550) for each visual task (visual acuity and contrast sensitivity) were analysed using the related-samples KruskalWallis test.

\section{RESULTS}

\section{Subjects}

The mean age of the speleologists was $40.65( \pm 10.93)$ years. The majority of the participants were males $(65.2 \%)$. The mean number of years of experience in caving was $15.20 \pm 11.20$ (median $=14.00$ ) years. Speleologists performed this activity during the daytime in approximately 12 days per year. The mean time spent in the cave was $4.30 \pm 2.49$ hours per day during the daytime. Accidents during this activity were reported by $21.7 \%$ of the participants. For the type of activity performed, the developed tasks consisted in: walking (vertical progression in galleries, sub-vertical and horizontal), topography, cartography, bats observation and photography.

\section{Visual function}

The majority of the subjects $(\mathrm{n}=13)$ have been observed in the last two years by an ophthalmologist (56.5\%), 7 subjects $(30.4 \%)$ had been observed at 4 years or more and 1 subject never had an ophthalmic observation. Optical correction was found in 14 subjects with 4 subjects using glasses just for near due to presbyopia.

We detected $26.1 \%(n=6)$ participants with visual impairment (decreased visual acuity) of which refractive error $(17.4 \%)$ was the major cause. Two subjects had a medical past history of a visual pathology, one had a retinal detachment and other had a keratoconus. The majority of the subjects had best uncorrected or corrected visual acuities LogMAR of 0.3 or better. Three subjects had a monocular visual acuity superior to LogMAR 0.3, two because of an uncorrected refractive error (both subjects reach LogMAR 0.2 with a pinhole) and one because of a keratoconus. One subject had also an intra-ocular pressure superior to $20 \mathrm{mmHg}$. There were no cases of manifest strabismus and the near convergence point was normal in all subjects $(<10 \mathrm{~cm})$.

The average values of contrast sensitivity were similar to the population norms (Table 1). There were not significant differences between the contrast sensitivity of the right (RE) and left eyes (LE) for the $3 \mathrm{cpd}(p=0.917), 6 \mathrm{cpd}(p=0.108), 12 \mathrm{cpd}(p=0.503)$ and $18 \mathrm{cpd}(p=0.634)$ spatial frequencies.

Binocular visual acuity in the cave environment was $-0.05 \pm 0.15$ LogMAR $(20 / 18)$ and all subjects had best uncorrected or corrected visual acuities LogMAR of 0.3 or better (Table 2). Only two participants had a reduced binocular visual acuity due to the presence of a refractive error.

All subjects had a normal near visual acuity of $1 \mathrm{M}$. Median value of stereoacuity was of 50 " in the lab and of $40^{\prime \prime}$ in the cave but the differences were not statistically significant ( $p=0.119)$.

During the cave activity blur vision was the most referred visual symptom $(62.5 \%, \mathrm{n}=10)$. However, in 3 subjects we found out that these symptoms could be related with the presence of a refractive error and 
Table 1. Monocular contrast sensitivity in log units measured in the laboratory.

\begin{tabular}{|l|c|c|c|c|c|c|c|c|c|c|c|c|}
\hline & \multicolumn{3}{|c|}{ Row A (3 cpd) } & \multicolumn{2}{c|}{ Row B (6 cpd) } & \multicolumn{2}{c|}{ Row C (12 cpd) } & \multicolumn{3}{c|}{ Row D (18 cpd) } \\
\cline { 2 - 14 } & RE & LE & $\mathbf{N}^{*}$ & RE & LE & N $^{*}$ & RE & LE & N*$^{*}$ & RE & LE & N $^{*}$ \\
\hline Mean & 1.69 & 1.69 & 1.61 & 1.85 & 1.91 & 1.66 & 1.64 & 1.55 & 1.08 & 1.13 & 1.10 & 0.56 \\
\hline Median & 1.63 & 1.78 & --- & 1.84 & 1.92 & --- & 1.54 & 1.69 & --- & 1.10 & 1.10 & --- \\
\hline Std. Deviation & 0.14 & 0.18 & 0.21 & 0.16 & 0.30 & 0.23 & 0.22 & 0.32 & 0.32 & 0.27 & 0.29 & 0.35 \\
\hline Minimum & 1.49 & 1.34 & --- & 1.55 & 0.91 & --- & 1.25 & 0.61 & --- & 0.47 & 0.47 & --- \\
\hline Maximum & 2.08 & 1.93 & --- & 2.29 & 2.29 & --- & 1.99 & 1.84 & --- & 1.55 & 1.55 & --- \\
\hline
\end{tabular}

*Population norms for age group 20-55 years of age in mesopic conditions.

Contrast sensitivity values are reported in log units.

$\mathrm{RE}$ - Right eye; LE - Left eye

Table 2. Monocular visual acuity and stereoacuity in the laboratory and binocular acuity and stereoacuity in the cave environment.

\begin{tabular}{|l|c|c|c|c|c|}
\hline & $\begin{array}{c}\text { Visual acuity* } \\
\text { in the lab RE }\end{array}$ & $\begin{array}{c}\text { Visual acuity* } \\
\text { in the lab LE }\end{array}$ & $\begin{array}{c}\text { Binocular acuity* } \\
\text { in the cave }\end{array}$ & $\begin{array}{c}\text { Stereoacuity } \\
\text { in the lab }\end{array}$ & $\begin{array}{c}\text { Stereoacuity } \\
\text { in the cave }\end{array}$ \\
\hline Mean & $0.13(20 / 27)$ & $0.04(20 / 22)$ & $-0.05(20 / 18)$ & 162.00 & 134.38 \\
\hline Median & $0.00(20 / 20)$ & $0.00(20 / 20)$ & $-0.10(20 / 16)$ & 50.00 & 40.00 \\
\hline Std. Deviation & 0.28 & 0.13 & 0.15 & 261.35 & 201.26 \\
\hline Minimum & $0.00(20 / 20)$ & $-0.10(20 / 16)$ & $-0.20(20 / 13)$ & 40.00 & 40.00 \\
\hline Maximum & $1.0(20 / 200)$ & $0.4(20 / 51)$ & $0.30(20 / 40)$ & 800.00 & 800.00 \\
\hline
\end{tabular}

*Visual acuities are reported in LogMAR units with the Snellen equivalent of the mean in parenthesis.

RE - Right eye; LE - Left eye

in 2 subjects we detected a past medical history of retinal detachment and keratoconus. The second most common symptom was visual irritability (43.8\%, $\mathrm{n}=7$ ). In this group one subject had a refractive error and other previous retinal detachment.

Binocular visual acuity for distance without filter was not statistically different from the visual acuity with the 550 or 450 filters $(p=0.093)$. Improved contrast sensitivity for the 4 spatial frequencies was observed with the use of $450 \mathrm{~nm}$ optical filters, but this difference was only statistically significant for the $6 \operatorname{cpd}(p=0.034)$ and for the $18 \operatorname{cpd}(p=0.026)$ spatial frequencies (Table 3$)$. For $3 \operatorname{cpd}(p=0.093)$ and $12 \operatorname{cpd}(p=0.368)$ spatial frequencies the differences were not statistically significant. Pairwise comparison for $6 \mathrm{cpd}$ and $18 \mathrm{cpd}$ spatial frequencies did not show significant differences between the three conditions (without filter, with 550 filters and 450 filters). However, speleologists preferred the $450 \mathrm{~nm}$ filters $(68.8 \%)$ when compared with the $550 \mathrm{~nm}$ filters $(6.3 \%)$ or without filter $(25.0 \%)$.

\section{Illuminance levels}

All vision tests in the laboratory were administered under artificial lighting mounted at ceiling level (21 fluorescent lamps with diffuser grilles, $18 \mathrm{~W}$ each, with a correlated color temperature of $4000 \mathrm{~K}$ and color rendering index of 82) with an illuminance ranging from 443 lux to 568 lux. For near vision tests, additional local lighting was used with a total illuminance ranging from 1552 lux on the lower plane to 2390 lux in the upper plane. In the cave environment the tests were administered under artificial lighting with an illuminance extending from 37 lux to 100 lux at $40 \mathrm{~cm}$ and at $2.5 \mathrm{~m}$, respectively. Daylight was not available either in the laboratory or in the cave.

In the cave environment the majority of the speleologists used a flashligh on the helmet or head at level 2 (26.1\%) without diffuser (47.8\%). In this position the mean illuminance values were $451.0 \pm$ 305.7 lux (Table 4).

Some speleologists used the headlamp with diffuser and only one used a hand flashlight, which had a very low value of illuminance (28 lux). The use of the diffuser filter resulted in lower illuminance values for all levels of light intensity ( 1 to 4 ) when comparing to headlamps without diffuser.

\section{DISCUSSION}

In the first part of the study (laboratory evaluation of visual performance) it was found a decreased monocular visual acuity in 6 speleologists, of which refractive error was the major cause. This finding must be analysed with caution, because in visual tasks involving detection of low contrast levels, a degraded visual acuity could have significant impact in terms of performance (Johnson \& Casson, 1995). Visual acuity screening for various occupations had been typically performed under near-optimal visual conditions (Johnson \& Casson, 1995). Nevertheless, many visual tasks are performed under low luminance or contrast.

We also observed two subjects with eye pathology (retinal detachment and keratoconus). These reported pathologies did not seem to be related with their caving activities. In this study, there were no apparent visual signs or symptoms of visual pathologies related to the exposure to the cave environment. This fact points to different work conditions compared to mines, which have an enormous impact on miners' health (Ovenseri-Ogbomo et al., 2012). Also, none of the speleologists had previous history of acute or chronic conjunctivitis, which leads the authors to conclude that occupationally associated eye diseases/disorders were not obvious among this group of speleologists. 
Table 3. Binocular visual acuity and contrast sensitivity in three conditions (without filter, with 550 filters and 450 filters).

\begin{tabular}{|c|c|c|c|}
\hline Visual function & $\begin{array}{c}\text { Mean } \pm \text { Stdd. } \\
\text { Deviation }\end{array}$ & Median & $\boldsymbol{p}$ \\
\hline Visual acuity without filter & $-0.05 \pm 0.15(20 / 18)$ & $-0.10(20 / 16)$ & \multirow{3}{*}{0.093} \\
\hline Visual acuity with 550 filter & $-0.04 \pm 0.16(20 / 18)$ & $-0.10(20 / 16)$ & \\
\hline Visual acuity with 450 filter & $-0.07 \pm 0.15(20 / 17)$ & $-0.10(20 / 16)$ & \\
\hline Contrast sensitivity $(3 \mathrm{cpd})$ without filter & $1.73 \pm 0.17$ & 1.63 & \multirow{3}{*}{0.104} \\
\hline Contrast sensitivity $(3 \mathrm{cpd})$ with 550 filter & $1.76 \pm 0.17$ & 1.78 & \\
\hline Contrast sensitivity $(3 \mathrm{cpd})$ with 450 filter & $1.84 \pm 0.12$ & 1.78 & \\
\hline Contrast sensitivity $(6 \mathrm{cpd})$ without filter & $1.99 \pm 0.24$ & 1.92 & \multirow{3}{*}{$0.034 *$} \\
\hline Contrast sensitivity $(6 \mathrm{cpd})$ with 550 filter & $1.92 \pm 0.16$ & 1.84 & \\
\hline Contrast sensitivity $(6 \mathrm{cpd})$ with 450 filter & $2.04 \pm 0.16$ & 2.07 & \\
\hline Contrast sensitivity $(12 \mathrm{cpd})$ without filter & $1.56 \pm 0.33$ & 1.54 & \multirow{3}{*}{0.368} \\
\hline Contrast sensitivity $(12 \mathrm{cpd})$ with 550 filter & $1.58 \pm 0.37$ & 1.62 & \\
\hline Contrast sensitivity $(12 \mathrm{cpd})$ with 450 filter & $1.64 \pm 0.39$ & 1.69 & \\
\hline Contrast sensitivity $(18 \mathrm{cpd})$ without filter & $1.05 \pm 0.34$ & 1.03 & \multirow{3}{*}{$0.026^{*}$} \\
\hline Contrast sensitivity $(18 \mathrm{cpd})$ with 550 filter & $0.97 \pm 0.25$ & 0.96 & \\
\hline Contrast sensitivity $(18 \mathrm{cpd})$ with 450 filter & $1.18 \pm 0.39$ & 1.25 & \\
\hline
\end{tabular}

Visual acuities are reported in LogMAR units with the Snellen equivalent of the mean in parenthesis. Contrast sensitivity values are reported in log units.

*Significant difference $(\mathrm{p}<0.05)$.

Table 4. Flashlight illuminance levels (lux).

\begin{tabular}{|l|c|c|c|c|c|c|c|}
\hline & $\begin{array}{c}\text { Flashlight } \\
\text { in leve1 1 } \\
\text { without } \\
\text { diffuser } \\
\text { (n=15) }\end{array}$ & $\begin{array}{c}\text { Flashlight } \\
\text { in leve1 2 } \\
\text { without } \\
\text { diffuser } \\
\text { (n=14) }\end{array}$ & $\begin{array}{c}\text { Flashlight } \\
\text { in leve1 3 } \\
\text { without } \\
\text { diffuser } \\
\text { (n=11) }\end{array}$ & $\begin{array}{c}\text { Flashlight } \\
\text { in leve1 4 } \\
\text { without } \\
\text { diffuser } \\
\text { (n=7) }\end{array}$ & $\begin{array}{c}\text { Flashlight } \\
\text { in leve1 } \\
\text { 1 with } \\
\text { diffuser } \\
\text { (n=3) }\end{array}$ & $\begin{array}{c}\text { Flashlight } \\
\text { in level } \\
\text { 2 with } \\
\text { diffuser } \\
\text { (n=2) }\end{array}$ & $\begin{array}{c}\text { Hand } \\
\text { flashlight } \\
\text { (n=1) }\end{array}$ \\
\hline Mean & 250.2 & 451.0 & 1551.3 & 1670.2 & 134.0 & 210.0 & 28.0 \\
\hline Median & 100.0 & 400.0 & 840.0 & 2000.0 & 170.0 & 210.0 & 28.0 \\
\hline $\begin{array}{l}\text { Std. } \\
\text { Deviation }\end{array}$ & 414.3 & 305.7 & 1165.9 & 1275.4 & 108.6 & 127.3 & --0 \\
\hline Minimum & 16.8 & 50.0 & 365.0 & 92.0 & 12.0 & 120.0 & 28.0 \\
\hline Maximum & 1600.0 & 1300.0 & 3000.0 & 4000.0 & 220.0 & 300.0 & 28.0 \\
\hline
\end{tabular}

The present study reports visual acuity and contrast sensitivity of speleologists performing activities in caves. In this environment, the binocular visual performance was not impaired. Binocular visual acuity in the cave was normal in the majority participants as well as binocular contrast sensitivity for all spatial frequencies. Only two subjects had binocular visual acuity of $>0.1$ LogMAR. These two subjects were not impaired in their work tasks, because visual acuity was sufficient for their activities (cave guide and bat observation). For example, a night security guard to recognize faces from a distance of $6 \mathrm{~m}$ under low illumination needs to have a visual acuity of 0.5 LogMAR (20/60) (Johnson \& Casson, 1995). The visibility conditions in the cave differ according to the level of light intensity used by cavers on their head/ helmet sources.

Binocular contrast sensitivity in the cave was normal for all spatial frequencies. It has been found that under mesopic (i.e., twilight) conditions, sensitivity to the lowest spatial frequencies is the same as that found under normal (photopic) conditions, but under scotopic (i.e., night-time) conditions, sensitivity functions are dramatically lowered across the entire spectrum from the normal contrast sensitivity function (Sekuler \& Blake, 1994). However, the findings of this study could be explained because of the light conditions used by cavers. All flashlights had a diffuser filter. However, cavers did not use it because the focused beam has increasingly higher values of illuminance from level 1 to 4 .

Visual acuity measurements were made in the cave with the speleologist in a static position and the helmet light set to a medium intensity $(451.0 \pm 305.7$ lux), which was the most commonly used by the cavers. Under this setting, the light conditions were very similar to a photopic environment, thus explaining the good visual performance in the cave. However, in some of the activities, like walking or during bats observation, the 
light conditions could be very low and similar to the scotopic environment.

The use of optical filters did not decreased visual acuity. The improvement in contrast sensitivity with the $450 \mathrm{~nm}$ filters could be beneficial to the cavers' activities. It is important that eye care practitioners are able to provide accurate advice on whether filters will provide a long-term benefit, prior to their recommendation to cavers.

Good lighting includes quantity and quality requirements, and should necessarily be appropriate to the activity/task being carried out, bearing in mind the comfort and visual efficiency of the worker (Piccoli et al., 2004; Pais \& Melo, 2011). According to the Artificial Light norm (DIN 5035-2, 1990), the level of illuminance that should be used for normal visual tasks with medium details is 500-750 lux, which is in accordance with most of the tasks performed by the cavers. For tasks with slight visual requirements and high contrast, 120-250 lux is required (analogy with the referred example of the mines) while for normal visual tasks with medium details the requirement is 500-750 lux. Visual demanding tasks with small details need illuminance values of 1000 to 1500 lux and very demanding with very small details visual tasks need levels of 2000-3000 lux (DIN 5035-2, 1990).

In this study, only 7 cavers had normalized illuminance values for the activities/task performed in the cave (headlamp without diffuser with 4 levels of intensity). Some of the cavers need to acquire appropriate equipment with adjustable lighting settings that are suitable for different activities, mainly for visual tasks with medium details.

\section{CONCLUSIONS}

This study was able to quantify the visual performance of speleologists in their natural environment of activity, the cave. There were no signs and symptoms of visual pathologies related to the cave low light exposure on these speleologists. Also, none of the cavers had previous history of acute or chronic conjunctivitis, which leads us to conclude that occupational associated eye diseases and disorders were not present among the speleologists. Most of the visual symptoms referred by the participants during the cave activity seemed to be related with the presence of a refractive error or a previous diagnosed ophthalmic pathology.

In the cave environment, binocular visual performance was not impaired by the existent lighting conditions (flashlights). The illuminance levels for the cavers who used the intensity light level 2 (without diffuser) on their headlamps were adequate to the majority of the activities/tasks performed.

The enhancement in contrast sensitivity when using the $450 \mathrm{~nm}$ filters could be beneficial to cavers or other researchers to potentially improve activities in the cave. Filters could be correlated with operational use after being tested in a larger sample and applied to different cave activities. Although this was an exploratory study, it is important to recognize the potential effects of using optical filters in activities that require medium to high detail observations (e.g., bat or mineral research). Further research is therefore needed to better understand the influence of lighting conditions in the visual symptoms for those carrying activities in caves. It is also important to observe the improvement in contrast sensitivity, when cavers or researchers spend longer time in the cave.

\section{ACKNOWLEDGEMENTS}

Thanks are due to the cavers who accepted to participate in this study. In addition, we would like to thank constructive comments of three anonymous reviewers, who have provided helpful comments to improve the content and focus of this paper.

Authors do not have any financial support or relationships that may pose conflict of interest.

\section{REFERENCES}

Blehm C., Vishnu S., Khattak A., Mitra S. \& Yee R.W., 2005 - Computer vision syndrome: a review. Survey of Ophthalmology, 50 (3): 253-262.

http://dx.doi.org/10.1016/j.

survophthal.2005.02.008

Boyce P.R., Beckstead J.W., Eklund N.H., Strobel R.W. \& Rea M.S., 1997 - Lighting the graveyard-shift: the influence of a daylight-simulating skylight on the task performance and mood of night-shift workers. Lighting Research and Technology, 29: 105-134.

http://dx.doi.org/10.1177/14771535970290030501

DIN 5035-2., 1990 - Artificial lighting, lighting of work places.

Eperjesi F., Fowler C.W. \& Evans B.J.W., 2002 - Do tinted lenses or filters improve visual performance in low vision? A review of the literature. Ophthalmic \& Physiological Optics, 22: 68-77.

http://dx.doi.org/10.1046/j.1475-1313.2002.00004.x

Friedman D.S., Repka M.X., Katz J., Giordano L., Ibironke J., Hawse P. \& Tielsch, J.M., 2009 - Prevalence of amblyopia and strabismus in white and African American children aged 6 through 71 months the Baltimore Pediatric Eye Disease Study. Ophthalmology, 116: $2128-2134$.

http://dx.doi.org/10.1016/j.ophtha.2009.04.034

Hudnell H.K., House D., Schmid J., Koltai D., Stopford W., Wilkins J., Savitz D.A., Swinker M. \& Music S., 2001 - Human visual function in the North Carolina clinical study on possible estuary-associated syndrome. Journal of Toxicology and Environmental Health, Part A, 62: 575-94.

http://dx.doi.org/10.1080/15287390151079633

Johnson C. \& Casson E., 1995 - Effects of luminance, contrast, and blur on visual acuity. Optometry and Vision Science, 72 (12): 864-869.

http://dx.doi.org/10.1097/00006324-199512000$\underline{00004}$

Kang I., Reem R.E., Kaczmarowski A.L. \& Malpeli J.G., 2009-Contrast sensitivity of cats and humans in scotopic and mesopic conditions. Journal of Neurophysiology, 102 (2): 831-840.

http://dx.doi.org/10.1152/jn.90641.2008

Kerkhof G.A., 1999 - Licht en prestatie. In: Symposium Lichten Gezondheid, SOLG, Amsterdam. 
Kohnen T., Bühren J., Kasper T. \& Terzi E., 2005 Quality of vision after refractive surgery. In: T. Kohnen $\&$ D. D. Koch (Eds.), Cataract and refractive surgery. Springer, Berlin, p. 303-314.

Ovenseri-Ogbomo G., Ocansey S., Abu E., Kyei S. \& Boadi-Kusi S., 2012 - Oculo-visual findings among industrial mine workers at Goldfields Ghana Limited, Tarkwa. Ophthalmology and Eye Diseases, 4: 35-42. http://dx.doi.org/10.4137/OED.S9204

Pais A. \& Melo R.B., 2011 - Lighting conditions in an office environment on visual comfort. In: Arezes J. S., Baptista M.P., Barroso P., Carneiro P., Cordeiro N., Costa R. \& Perestrelo G.P. (Eds.), Proceedings of the International Symposium on Occupational Safety and Hygiene, Guimarães, Sociedade Portuguesa de Segurança e Higiene Ocupacionais, p. 456-459 .

Piccoli B., Soci G., Zambelli P. \& Pisanello D., 2004 Photometry in the workplace: The rationale for a new method. The Annals of Occupational Hygiene, 48 (1): 29-38. http://dx.doi.org/10.1093/annhyg/meg076

Reinhold K. \& Tint P., 2009 - Lighting of workplaces and health risks. Electronics and Electrical Engineering, 2 (90): 11-14.

Reyes M., Gallagher S. \& Sammarco J.J., 2013 Evaluation of visual performance when using inc andescent, fluorescent, and LED machine lights in mesopic conditions. IEEE Transactions of the Industry Applications Society, 49 (5).

Sekuler R. \& Blake R., 1994 - Perception (3 $3^{\text {rd }}$ ed.). McGraw Hill, New York.
Stockman A. \& Sharpe L.T., 2006 - Into the twilight zone: the complexities of mesopic vision and luminous efficiency. Ophthalmic \& Physiological Optics, 26: 225-239. http://dx.doi.org/10.1111/j.1475-1313.2006.00325.x

Thomas R.S., Wright S.T., Clark P.J., Thompson W.T. \& Gooch J.M., 2010 - Optical filter effects on night vision goggle acuity and preservation of dark adaptation. Aviation, Space, and Environmental Medicine, 81 (9): 869-872. http://dx.doi.org/10.3357/ASEM.2644.2010

Van Bommel W.J.M., 2006 - Non-visual biological effect of lighting and the practical meaning for lighting for work. Applied Ergonomics, 37: 461-466. http://dx.doi.org/10.1016/j.apergo.2006.04.009

Veitch J., 2001 - Psychological processes influencing lighting quality. Journal of the Illuminating Engineering Society, 30 (1): 124-140. http://dx.doi.org/10.1080/00994480.2001.10748341

Wood J.M. \& Owens D.A., 2005 - Standard measures of visual acuity do not predict drivers' recognition performance under day or night conditions. Optometry and Vision Science, 82: 698-705.

http://dx.doi.org/10.1097/01. opx.0000175562.27101.51

Woodside G. \& Kocurek K., 1997 - Environmental safety and health engineering. John Wiley \& Sons Inc., New York.

Zavod M., 2004 - The effects of stimulus motion on contrast sensitivity: dynamic sensitivity functions. PhD dissertation, University of Central Florida, Orlando. 